\title{
Gas-Flow Resistance in Continuous Macropores in Silica Rods Prepared by Freezing Transitional Structures of Phase Separation
}

\author{
Ryoji TAKAHASHI, Satoshi SATO, Toshiaki SODESAWA, Akihiro HAGA and Hironobu NISHINO \\ Department of Materials Technology, Faculty of Engineering, Chiba University, Yayoi-cho, Inage-ku, Chiba-shi 263-8522
}

\author{
相分離の過渡構造を凍結して作製した連続貫通マクロ孔を \\ 有するシリカロッド中のガス流動特性 \\ 高橋亮治·佐藤智司 · 袖澤利昭 · 芳賀昌宏 · 西野弘展 \\ 千葉大学工学部物質工学科, 263-8522 千葉市稲毛区弥生町 1-33
}

\begin{abstract}
Pressure loss in gas flow in three-dimensionally interconnected macropores in silica gel rods was investigated. In the preparation of the silica gel, the continuous macropores are formed by inducing phase separation in a solution containing tetraethoxysilane (TEOS) and poly (ethylene oxide) (PEO), and subsequent freezing of its transitional structures by gelation. Average macropore size of the silica gels was systematically controlled from 25 to $0.6 \mu \mathrm{m}$ by changing PEO content in the solution without affecting porous morphology. The pressure loss in gas flow in the continuous macropores can be approximated with a simple straight channels model, and well reproduced with the Hargen-Poiseuille's equation without any correction, in contrast to that in columns packed with micrometer-size particles, which show substantial higher pressure loss than the continuous macropores. The low flow resistance in the macroporous silica rods would be attributed to the absence of necks in flow pathways.
\end{abstract}

[Received September 10, 2003; Accepted November 27, 2003]

Key-words: Gas-flow resistance, Silica gel, Macroporous material, Phase separation, Hargen-Poiseuille

\section{Introduction}

Porous materials have been widely used as adsorbents, filters, catalyst supports and so on. The characteristics of porous materials are described with structural parameters of pores such as specific surface area, porosity, average pore size and pore size distribution. In the above applications, however, molecular transportation in the pores sometimes governs overall performances of the porous materials. In small pores categorized in mesopore, typically smaller than $50 \mathrm{~nm}$, material transportation proceeds mainly via Knudsen diffusion, and diffusion coefficient of gas molecules, $D_{\mathrm{k}}$, linearly decreases with decreasing pore size. ${ }^{1)}$

$$
D_{\mathrm{k}}=\frac{2}{3} r_{\mathrm{p}}\left(\frac{8 R T}{\pi M}\right)^{1 / 2}
$$

where $r_{\mathrm{p}}$ is pore radius, $R$ : gas constant, $T$ : temperature, and $M$ : molecular weight of gas molecules. In micropores, where the pore size and molecular size are comparable, gas diffusion is substantially restricted. Therefore, bimodal porosity with macropores and mesopores or micropores has great advantage in gas-phase diffusion in porous materials. Molecular transportation in macropores, larger than $50 \mathrm{~nm}$, can also proceed via mass flow in addition to the diffusion under some conditions.

Since gas-flow resistance in micrometer-restricted spaces is affected by various structural parameters such as size of flow pathway and the number and nature of the curves, bends, branches, necks and so on, gas-flow characteristics in macropores has been described with complex equations which were derived from some structural models. ${ }^{2,3)}$ In the equations, some variable parameters whose physical meaning is not always clear are introduced, and make difficult to understand gas-flow property in macropores. The difficulties also present in sample preparation for the measurement of gas-flow resistance. If we intend to deal with only the effect of pore size, for example, we have to prepare several macroporous samples in which porous morphology except for macropore size is exactly the same among the samples. However, it was very difficult to serve such samples by classical techniques.
On the other hand, recent advantage in sol-gel science and technology makes it possible to provide three-dimensionally interconnected macropores with varied size in a monolithic silica gel. ${ }^{4)-7)}$ High connectivity in macropores without neck and high uniformity in macropore size are the characteristics of the material, and the pore size in a silica gel can be controlled typically from 0.2 to $50 \mu \mathrm{m}$ without affecting the morphology. That is, only the macropore size varies in a self-similar manner in the monolithic silica gel. The macroporous silica, therefore, would be a good model to consider the gas flow resistance in macroporous systems with varied pore size. In addition, the macroporous silica gel also has mesopores whose size is controllable. We recently applied the silica to solid catalysts, such as silica-supported nickel, ${ }^{8}$ silica-zirconia, ${ }^{9), 10)}$ and silica-alumina. ${ }^{11), 12)}$ In their use as monolithic catalysts, it is necessary to understand the gas flow nature in the macropores. In this work, we have prepared silica gel rods with continuous macropores whose size is varied from 0.6 to $25 \mu \mathrm{m}$, and have evaluated gas-flow resistance in the macropores.

\section{Experimental}

2.1 Preparation of macroporous silica gel rods

The silica gel rods were prepared from tetraethoxysilane (TEOS, Shin-Etsu Chemical Co.) and polyethylene oxide with average molecular weight of 100000 (PEO, Aldrich). A $14 \mathrm{~cm}^{3}$ of TEOS was added into $17.6 \mathrm{~cm}^{3}$ of $1 \mathrm{~mol} \cdot \mathrm{dm}^{-3}$ nitric acid aqueous solution containing PEO. ${ }^{6)}$ The PEO content was varied from 0.6 to $2.0 \mathrm{~g}$. Although the mixed solution was initially inhomogeneous, it became clear after stirring for several minutes. Then, the solution was poured into a polystyrene tube with inner diameter of $8 \mathrm{~mm}$, sealed tightly, and held at $50^{\circ} \mathrm{C}$ for $24 \mathrm{~h}$ for gelation. The obtained wet gel with rod-shape was extracted from the tube, and slowly dried at $50^{\circ} \mathrm{C}$ to avoid generation of crack in the gel body, followed by calcination at $800^{\circ} \mathrm{C}$ for $2 \mathrm{~h}$. The diameter of thus prepared silica gel rods was ca. $6 \mathrm{~mm}$.

\subsection{Characterization}

Scanning electron microscopes (SEM; SM200 and ABT32T, 
Topcom) were employed to observe micrometer-scale morphology of a fractured surface of the obtained silica gel. Nitrogen adsorption isotherm for each sample was measured with automated adsorption apparatus (Omnisorp 100CX, Coluter) at $-196^{\circ} \mathrm{C}$. Total mesopore volume, $V_{\text {meso }}$, was calculated from volume of adsorbed nitrogen at $P / P_{0}>0.95$. The volume fraction of macropores, $\varepsilon$, is calculated according to the following equation, where $V_{\text {bulk }}$ and $V_{\text {silica }}$ are bulk and silica skeleton volumes, respectively.

$$
\varepsilon=\frac{V_{\text {bulk }}-V_{\text {silica }}-V_{\text {meso }}}{V_{\text {bulk }}}
$$

The value of $V_{\text {bulk }}$ was obtained from length and diameter of the silica rod, and that of $V_{\text {silica }}$ was calculated from weight of the silica rod and density of a silica glass, $2.2 \mathrm{~g} \cdot \mathrm{cm}^{-3}$.

2.3 Measurement of pressure loss in gas flow in macropores

For the experiment of gas-flow resistance, the silica gel rod was cut into an appropriate length, typically ca. $1 \mathrm{~cm}$, and both surfaces were grinded to be parallel. Figure 1 illustrates the apparatus for the measurement. The silica rod cut and grinded was jointed with two glass pipes using a thermally-shrinkable tube made with silicone rubber. Then, it was set in a Z-module (Waters), which was originally used in HPLC measurement, and pressured over the thermally-shrinkable tube from sectional direction to avoid bypath flow and leak during the measurement of the pressure loss. For the measurement of the pressure loss, either mercury or water manometer was set to observe the difference in pressure between the entrance and the exit. The relation between pressure loss, $\Delta P$, and volumetric flow rate of air at the exit, $Q_{\mathrm{e}}$, was monitored. In the gas flow, gas volume changes in the rod due to the pressure gradient. Therefore, the $Q_{\mathrm{e}}$ was corrected to obtain a real flow rate, $Q .^{13)}$

$$
Q=\frac{3\left[\left(P_{1} / P_{2}\right)^{2}-1\right]}{2\left[\left(P_{1} / P_{2}\right)^{3}-1\right]} \times Q_{\mathrm{e}}
$$

Where $P_{1}$ and $P_{2}$ are the pressure at the entrance and the exit, respectively. Because the samples contain only small mesopores, $<5 \mathrm{~nm}$, gas flow in mesopores can be neglected. Then, average linear velocity of the air, $u$, in macropores in the silica rod was calculated from $Q, \varepsilon$, and diameter of the rod, $d_{\text {rod }}$, according to

$$
u=\frac{Q}{\varepsilon \times \pi\left(d_{\mathrm{rod}} / 2\right)^{2}}
$$

As a comparison, particles-packed columns made with commercial silica gel beads (Wakogel, Wako Pure Chemical Ind.) with various diameters and commercial silica-alumina pellet (N631L, Nikki Chemical Co.) were also served for the experiment of gas-flow resistance. The measurement of the relation between $\Delta P$ and $Q$ for the N631L pellet was carried out in a similar man-

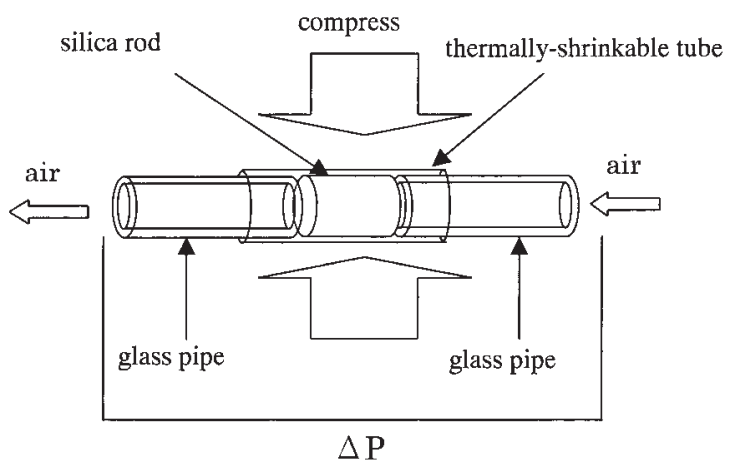

Fig. 1. Illustration of apparatus for the measurement of gas-flow resistance. ner as performed for macroporous silica gel rods. For the measurement of the packed column, a bed of particles with the length of ca. $1 \mathrm{~cm}$ was made in a glass tube with inner diameter of $4 \mathrm{~mm}$ using a glass wool. The $\Delta P$ in the bed was obtained by subtracting the blank value preliminarily measured for glass wool from the measured value. For the calculation of $u$, inner diameter of the glass tube was used instead of $d_{\text {rod }}$ in Eq. (2). The pressure loss in straight pipes with inner diameter of 4.0, 2.0, 0.53 and $0.10 \mathrm{~mm}$ was also measured for comparison.

\section{Results and discussion}

3.1 Preparation and characterization of macroporous silica gel

Figure 2 shows SEM photographs of fractured surfaces of the obtained silica gel rods. Continuous micrometer-size pores are observed in all the samples. The pore size is uniform in each sample, while it varies among the samples. According to Nakanishi and coworkers, the macropores are formed when transitional structures of phase separation are fixed by gelation of silica phase. ${ }^{7)}$ The incorporation of PEO in the sol-gel solution has an effect to increase the mixing free energy in the solution, i.e. to destabilize the homogeneous state. Then, phase separation proceeds during sol-gel reaction at appropriate conditions. The phase rich in silica becomes mesoporous silica skeleton, while the other phase becomes macropores after drying. In a polymer solution, phase separation usually proceeds via spinodal decomposition, where bicontinuous morphology grows in a self-similar manner with the time elapse. Therefore, a gel with small macropores is obtained when the gelation of silica occurs just after the onset of phase separation (Fig. 2(F)). On the other hand, a gel with large macropores is obtained when the gelation of silica occurs after the domains grow enough (Fig. 2(A)). Because of self-similarity in the growing morphology in spinodal decomposition, only the size of pores can be varied without affecting pore connectivity. Table 1 lists average pore sizes, $d_{\text {pore }}$, which are the average of 10-20 times measurement at random positions in the photos. The pore size sequentially decreases from 25 to $0.65 \mu \mathrm{m}$ with increasing PEO content from 0.8 to $1.8 \mathrm{~g}$. At the PEO content of $0.6 \mathrm{~g}$, a gel monolith with macropores could not be obtained because silica phase precipitates before gelation. At the PEO content of $2.0 \mathrm{~g}$, gel with macropores could not be obtained either, because gelation occurs before onset of phase separation.

Figure 3 shows a nitrogen adsorption isotherm at $-196^{\circ} \mathrm{C}$ for PSO-12 in Table 1. If the sample had only macropores observed with SEM, its BET surface area must be as small as ca. $2 \mathrm{~m}^{2} \cdot \mathrm{g}^{-1}$ according to simple geometrical calculation. The large BET surface area over $400 \mathrm{~m}^{2} \cdot \mathrm{g}^{-1}$ ensures us the presence of nanometer-size pores in the silica skeleton. Judging from the Langmuirtype isotherm, the size of pores is considered to be smaller than $3 \mathrm{~nm}$. In fact, no peak was observed in the nanometer-scale pore size distribution curve calculated from the isotherm (not shown). Although the volume of the nanometer-size pores varies among the samples slightly, the difference is as small as $10 \%$. The volume fraction of macropores, $\varepsilon$, calculated from Eq. (2) varied from 0.52 to 0.54 among the samples.

In Table $2, \varepsilon$ of columns made with beads with different size was listed. The $\varepsilon$ for the closed packing of monodispersed spheres is 0.26 , and that for the simple cubic arrangement is 0.48 . For the particles with size of $10 \mu \mathrm{m}$, the packing density is the same as that of simple cubic. The particles with size $>30 \mu \mathrm{m}$ can be packed denser than simple cubic, while packing of particles with size of $5 \mu \mathrm{m}$ is very loose. The value of $\varepsilon$ for $5 \mu \mathrm{m}$ particles is even larger than those of macroporous silica rods. On the other hand, the N631L pellet has little macropores although it is composed of large particles (Fig. 4). Macroporous structure cannot be devel- 

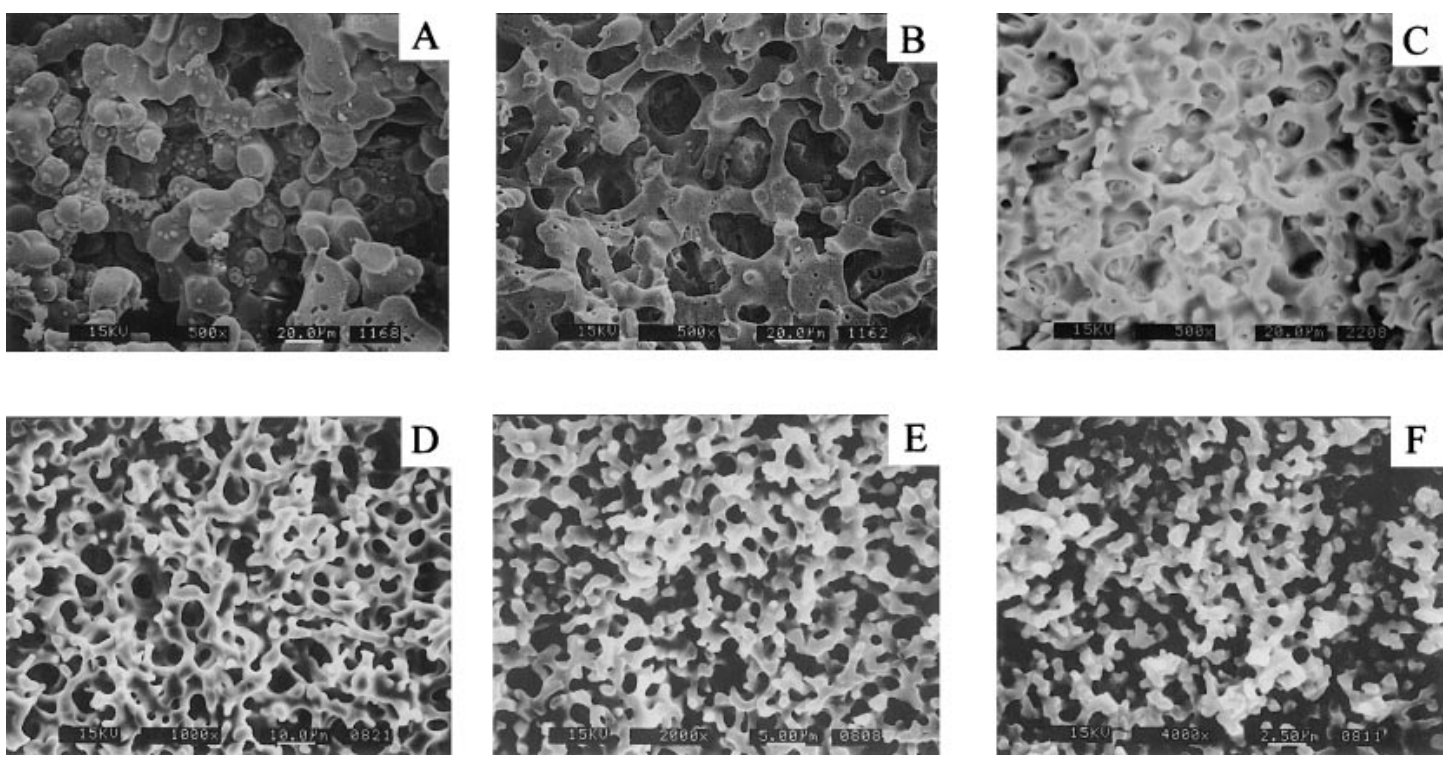

Fig. 2. SEM micrographs for fractured surfaces of macroporous silica gel rods. (A) POS-08, (B) POS-10, (C) POS-12, (D) POS-14, (E) POS-16, (F) POS-18. Note that the length of a scale bar is the same for (A) to (C), while decreases sequentially from (C) to (F). The porous morphology seems to be the same, and only the size varies.

Table 1. Gas-Flow Properties in Macroporous Silica Rods

\begin{tabular}{cccc}
\hline Sample & PEO $/ \mathrm{g}$ & $d_{\text {pore }} / \mu \mathrm{m}$ & $\Delta P / u L / \mathrm{Pa} \mathrm{s} \mathrm{m}^{-2}$ \\
\hline POS-08 & 0.80 & 25 & $9.13 \times 10^{5}$ \\
POS-10 & 1.00 & 18 & $1.94 \times 10^{6}$ \\
POS-12 & 1.20 & 8.0 & $8.72 \times 10^{6}$ \\
POS-14 & 1.40 & 4.5 & $3.15 \times 10^{7}$ \\
POS-16 & 1.60 & 1.4 & $2.35 \times 10^{8}$ \\
POS-18 & 1.80 & 0.65 & $1.78 \times 10^{9}$ \\
\hline
\end{tabular}

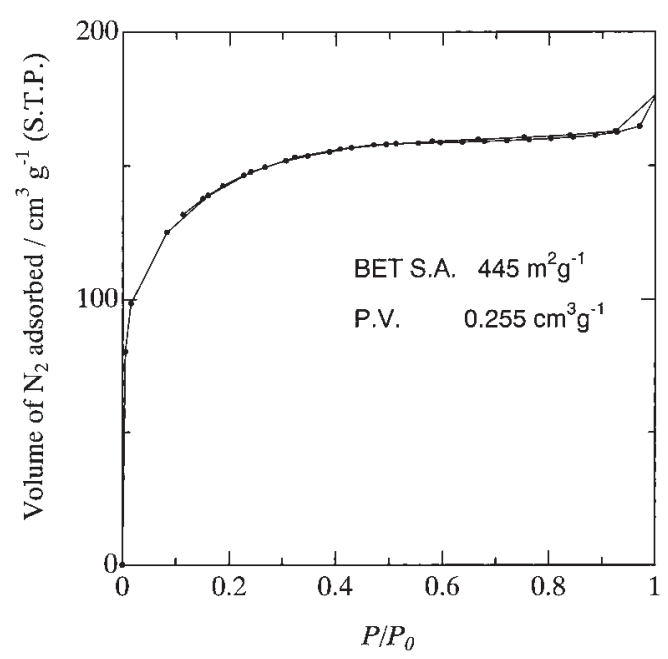

Fig. 3. $\mathrm{N}_{2}$ adsorption isotherm for POS- 12 at $-196^{\circ} \mathrm{C}$.

oped in the N631L pellet probably because of the restriction in the preparation technique.

3.2 Gas flow in continuous macropores

Figure 5 shows the dependence of pressure loss per unit length,
Table 2. Gas-Flow Properties in Columns Packed with Silica Particles with Varied Size and in Commercial Catalyst Pellet, N631L

\begin{tabular}{ccccc}
\hline Sample & $P S / \mu \mathrm{m}^{\mathrm{a}}$ & $\varepsilon$ & $\Delta P / u L / \mathrm{Pa} \mathrm{s} \mathrm{m}^{-2}$ & $P S_{\text {call }} / \mu \mathrm{m}^{\mathrm{b}}$ \\
\hline C200 & $75-150$ & 0.43 & $1.88 \times 10^{6}$ & 88 \\
LC50 & 50 & 0.45 & $4.76 \times 10^{6}$ & 52 \\
LC30 & 30 & 0.43 & $9.44 \times 10^{6}$ & 39 \\
LC10 & 10 & 0.48 & $4.65 \times 10^{7}$ & 15 \\
LC5 & 5 & 0.56 & $1.33 \times 10^{8}$ & 7.3 \\
N631L & 26 & 0.14 & $3.2 \times 10^{9}$ & 7.1 \\
\hline
\end{tabular}

${ }^{\text {a }}$ Size of particles packed in column.

${ }^{\mathrm{b}}$ Size of particles calculated from the pressure loss using Kozeny-Carman equation.

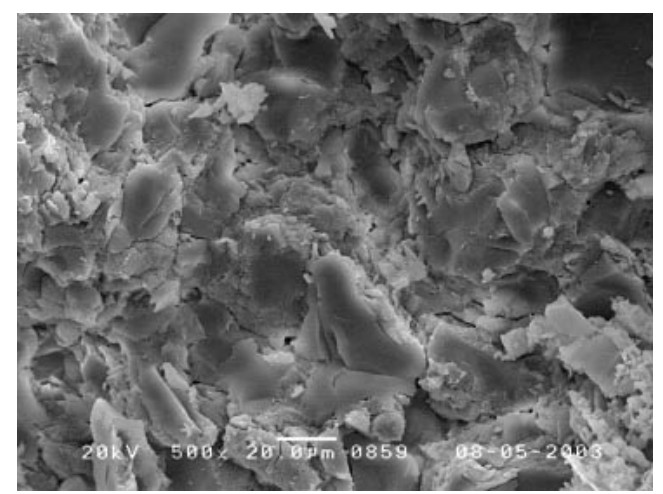

Fig. 4. SEM micrograph for fractured surface of commercial catalyst pellet, N631L.

$\Delta P / L$, on the average linear velocity of air in macropores, $u$, when air is flowed in the macropores of the silica rods. Good linearity was observed for all the samples while the slope increases with the decrease in the macropore size. Here, the Reynolds number, $R e=d_{\text {pore }} u \rho / \mu$, in gas flow is calculated to be ca. 6 for the largest, where $\rho$ and $\mu$ are density and viscosity of air, respectively. The slope, $\Delta P / u L$, for each sample obtained by the least square 

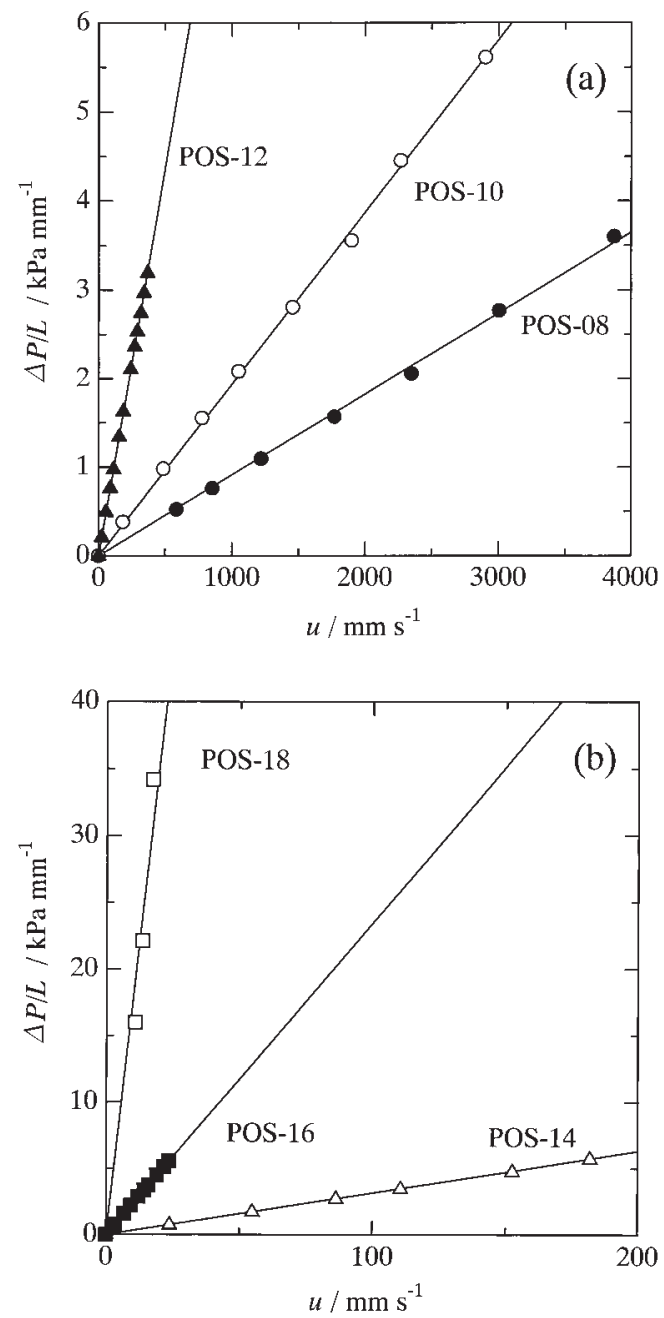

Fig. 5. Dependence of pressure loss on flow rate of air in macroporous silica gel rods.

fitting is regarded as normalized pressure loss, and is listed in Table 1. In the fitting, the correlation factor exceeded 0.999 for all the samples, and the difference in the values for the different rods of the same sample was smaller than $2 \%$. The value of $\Delta P / u L$ drastically increases with the decrease in macropore size. For the sample with the smallest macropores, POS $-18, \Delta P / u L$ is about 1000 times larger than for the sample with the largest macropores, POS-08.

Values of $\Delta P / u L$ for particles-packed columns and for a commercial catalyst pellet, N631L, are listed in Table 2 together with $\varepsilon$. In the packed columns, a linear correlation between $\Delta P / L$ and $u$ is also observed, and $\Delta P / u L$ increases with the decrease in the size of the particles. The reproducibility in the measurement for the packed columns was lower than that for the macroporous silica rods, probably because the packing state of the particles would slightly varies among the columns. The N631L pellet shows very large $\Delta P / u L$.

Figure 6 summarizes the relation between $\Delta P / u L$ and $d_{\text {pore }}$ in double logarithmic scales. Here, the particle sizes are used instead of $d_{\text {pore }}$ for the reference packed columns and N631L pellet. $\Delta P / u L$ linearly decreases with increasing $d_{\text {pore }}$ for both macroporous silica rods and packed columns. At the fixed $d_{\text {pore }}$, however, $\Delta P / u L$ for macroporous silica rods is always smaller than those of the packed columns. The low $\Delta P / u L$ for macroporous silica rods suggests that gas in their pores flows very smoothly. Concerning the slope between $\Delta P / u L$ and $d_{\text {pore }}$, data points for macroporous

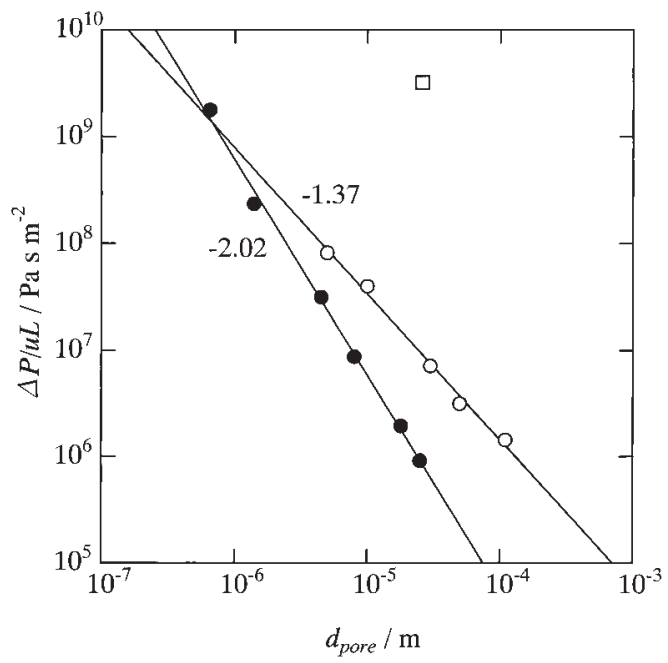

Fig. 6. Double logarithmic plots of the relation between $\Delta P / u L$ and pore size, $d_{\text {pore }}$. Closed circles are the data points for macroporous silica gel rods, open circles are packed columns, and an open square is N631L pellet. For the packed columns and N631L pellet, particle size is used instead of $d_{\text {pore }}$ for the convenience in the comparison.

silica rods and packed columns are on straight lines with slopes of -2.02 and -1.37 , respectively, although the unevenness in the latter case is relatively large. For the packed columns, not only the particle size but also the packing density varies among the samples ( $\varepsilon$ in Table 2), so that the slope of -1.37 would have no physical meaning. The interpretation of the pressure loss in the packed columns will be further considered in the following section.

On the other hand, $\varepsilon$ as well as pore connectivity among macroporous silica rods can be regarded as constant, and contribution of parameters other than size is considered to be negligible in the relation between $\Delta P / u L$ and $d_{\text {pore }}$. The Hargen-Poiseuille's equation is known as the simplest formulation for laminar flow at $\operatorname{Re}<2300 .^{3)}$

$$
\Delta P=\frac{32 \mu u L}{d_{\text {pore }}^{2}}
$$

In the equation, $\Delta P / u L$ is shown to be proportional to $d_{\text {pore }}^{-2}$. The slope of -2.02 in the double logarithmic plot in Fig. 6 suggests that the morphology of the gas-flow pathway in the macroporous silica rods does not change among the samples, and that only the size of pores changes in a self-similar manner.

The morphology of the macroporous silica rods can be described as superposition of infinite number of trigonometric functions oriented randomly in a three-dimensional space. This structure has no neck, and is composed of linked smooth curves. Therefore, the gas flow in the macropores is considered to be close to that in straight pipes. Then, $\Delta P / u L$ in straight pipes were measured for

Table 3. Gas-Flow Resistance in Straight Pipes

\begin{tabular}{cc}
\hline$d / \mathrm{mm}$ & $\Delta P / u L / \mathrm{Pa} \mathrm{s} \mathrm{m}^{-2}$ \\
\hline 4.0 & $3.62 \times 10^{1}$ \\
2.0 & $1.44 \times 10^{2}$ \\
0.53 & $2.25 \times 10^{3}$ \\
0.10 & $6.06 \times 10^{4}$ \\
\hline
\end{tabular}




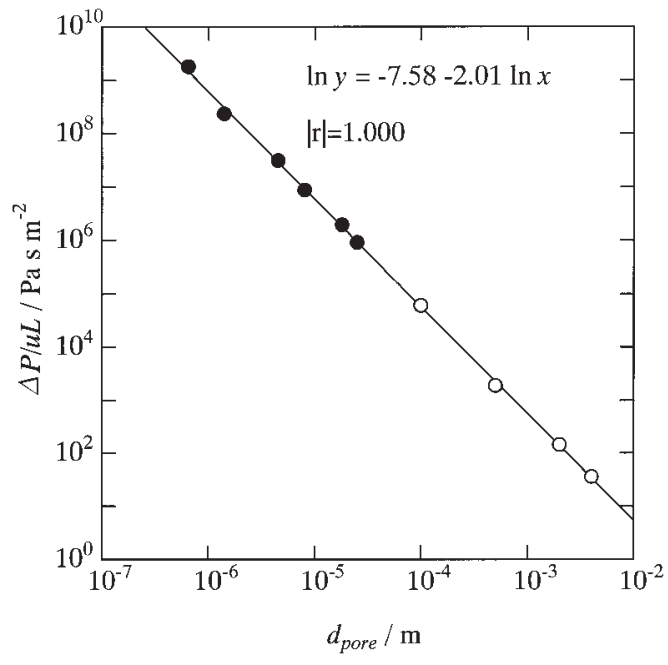

Fig. 7. Double logarithmic plots of the relation between $\Delta P / u L$ and pore size, $d_{\text {pore }}$. Closed circles are the data points for macroporous silica gel rods, and open circles are straight pipes.

comparison as summarized in Table 3. Figure 7 shows the relation between $\Delta P / u L$ and $d_{\text {pore }}$ in double logarithmic scales for both the straight pipes and the macroporous silica gel rods. The data points for both series are on the same straight line with a slope of -2.01 . This means that the macropores formed by fixing a transitional structure of phase separation can be approximated as a bundle of straight pipes with the same size as macropores concerning the gas flow. In fact, the viscosity of air estimated from $Y$ intercept $(-7.58)$ of the fitting line using Hargen-Poiseuille's equation $\left(1.6 \times 10^{-5} \mathrm{~kg} \cdot \mathrm{m}^{-1} \cdot \mathrm{s}^{-1}\right)$, agrees with the real viscosity of air at room temperature $\left(1.81 \times 10^{-5} \mathrm{~kg} \cdot \mathrm{m}^{-1} \cdot \mathrm{s}^{-1}\right)$. Strictly speaking, the length of flow pathway in the macroporous silica rods is somewhat extended from the length of the rods by the presence of curves. However, this effect would be negligible compared with other factors such as experimental errors in the estimation of macropore size and the expanse in pore size distribution. Therefore, the gas flow resistance in macropores with uniform size without neck can be approximated with simple straight channels model, and is represented with the simple Hargen-Poiseuille's equation.

3.3 Comparison of gas-flow resistance between silica rods and packed columns

The difference between the pressure loss in macroporous silica rods and in packed columns at the same pore size (Fig. 6) would mainly come from the difference in the geometry of the pathway for the gas flow. In the data processing for the packed columns, the Kozeny-Carman equation, which is derived from HargenPoiseuille's equation, has been widely adopted. ${ }^{3)}$

$$
\Delta P=2 k \mu u L \frac{(1-\varepsilon)^{2}}{\varepsilon^{2}} S_{\mathrm{v}}^{2}
$$

Where $S_{\mathrm{v}}$ is an external surface area of particles per unit volume, and $k$ is an empirical parameter relating to the extended length of the flow pathway in the column and usually 2.5 is used. For spherical particles, $S_{\mathrm{v}}$ is expressed with particle size, $P S$, as $S_{\mathrm{v}}=6 / P S$. Then, we can calculate particle size from the pressure loss data according to the Eq. (6). The calculated particle sizes are close to the real particle sizes as shown in Table 2.
For the straight pipes, $S_{\mathrm{v}}=4 / d_{\text {pore }}$ and $k=1$, resulting in Hargen-Poiseuille's equation. The product of $(6 / 4)^{2}$ by $k$ for the packed column is about 5.6, and can be regarded as the additional pressure loss for the packed column compared to the straight pipes. Because the macropores in the silica rod can be regarded as a bundle of straight pipes with the size of macropores as described in the previous section, the pressure loss in the silica rods can be predicted to be smaller by the factor of 5.6 than that in the packed columns with the same porosity and the same size. This prediction well explains the present results in Fig. 6.

In the evaluation of the rod-type monolithic silica gel as a HPLC column, Minakuchi and coworkers reported that the pressure loss in the rod silica during the flow of a liquid phase was 6-8 times smaller than that in the packed column. ${ }^{14)}$ Taking into account the low $\varepsilon$ in the commercial packed HPLC column, their results essentially agree with the above discussion, and our results for the gas flow. Thus, it is suggested that monolithic macroporous silica gel prepared by freezing transitional structures of phase separation has great advantage in practical use compared with other macroporous systems.

\section{Conclusion}

Silica gel rods with three dimensionally interconnected macropores were prepared by freezing transitional structures of phase separation, and the dependence of pressure loss in gas flow in the macropores on the pore size was investigated. The pressure loss in the gas flow in the macropores can be approximated with a simple straight channels model, and well reproduced with the Hargen-Poiseuille's equation without any correction. The absence of neck structure in the macroporous morphology is suggested to be the origin of this low-pressure loss compared with other macroporous systems.

\section{References}

1) Smith, J. M., "Chemical Engineering Kinetics, 3rd ed.," McGraw-Hill, New York (1981) pp. 452-461.

2) Richardson, J. T., Peng, Y. and Remue, D., Appl. Catal. A: General, Vol. 204, pp. 19-32 (2000).

3) Yoshio, O., "Porous Materials-Characterization, Production and Application-," Ed. by Takeuchi, Y., Fuji Technosystem, Tokyo (1999) pp. 289-291 [in Japanese].

4) Nakanishi, K. and Soga, N., J. Am. Ceram. Soc., Vol. 74, pp. 2518-2530 (1991).

5) Nakanishi, K. and Soga, N., J. Non-Cryst. Solids, Vol. 139, pp. 1-13 (1992).

6) Nakanishi, K., Komura, H., Takahashi, R. and Soga, N., Bull. Chem. Soc. Jpn., Vol. 67, pp. 1327-1335 (1994).

7) Nakanishi, K., J. Porous Materials, Vol. 4, pp. 67-112 (1997).

8) Nakamura, N., Takahashi, R., Sato, S., Sodesawa, T. and Yoshida, S., Phys. Chem. Chem. Phys., Vol. 2, pp. 4983-4990 (2000).

9) Takahashi, R., Nakanishi, K. and Soga, N., J. Ceram. Soc. Japan, Vol. 106, pp. 772-777 (1998).

10) Takahashi, R., Sato, S., Sodesawa, T., Suzuki, K., Tafu, M., Nakanishi, K. and Soga, N., J. Am. Ceram. Soc., Vol. 84, pp. 1968-1976 (2001).

11) Takahashi, R., Sato, S., Sodesawa, T. and Yabuki, M., J. Catal., Vol. 200, pp. 197-202 (2001).

12) Yabuki, M., Takahashi, R., Sato, S., Sodesawa, T. and Ogura, K., Phys. Chem. Chem. Phys., Vol. 4, pp. 4830-4837 (2002).

13) Kojima, T., Ooi, N. and Morishita, F., "Method of Gas Chromatograph,” Kyoritsu Shuppan (1985) pp. 14-14 [in Japanese].

14) Minakuchi, H., Nakanishi, K., Soga, N., Ishizuka, N. and Tanaka, N., J. Chromatogr. A, Vol. 797, pp. 121-131 (1998). 